\title{
INTRODUCTION
}

The U.S. Navy has conducted a limited flight test evaluation of the F/A-18 HARV, the purpose of which was to provide in-flight validation of extensive ground-based simulation, assessment of overall handling qualities at high angle of attack (AOA), and determination of tactical utility benefits generated from the aircraft's thrust vectoring system. This evaluation was part of a joint NASANNavy study which was initiated to quantify high AOA control power requirements under the NASA High Angle of Attack Technology Program (HATP). Currently, flying qualities specifications define control power requirements qualitatively. As tactical aircraft continue to push the envelope in terms of high AOA maneuverability, control power becomes an increasingly important design driver.

This presentation will highlight results from the latest Navy evaluation of the HARV (March 1994) and focus primarily on the impressions from a piloting standpoint of the tactical utility of thrust vectoring. Issues to be addressed will be mission suitability of high AOA flight, visual and motion feedback cues associated with operating at high AOA, and the adaptability of a pilot to effectively use the increased control power provided by the thrust vectoring system.

\section{Navy And The HARV: High Angle Of Attack Tactical Utility Issues}

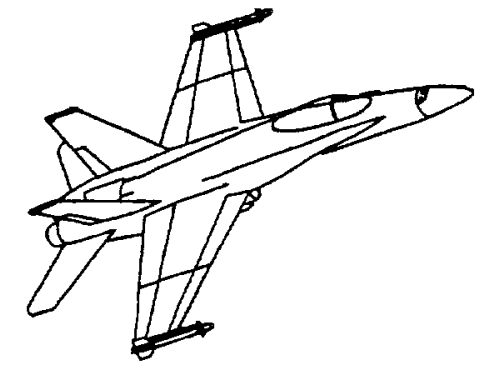

LT C. A. Sternberg, USN Capt Ricardo Traven, CAF Mr. James Lackey

Naval Air Warfare Center Patuxent River, Maryland 


\section{BACKGROUND}

This was the second of two evaluations conducted by the U.S. Navy, the first being in November of 1992. Unique to these evaluations was the operational F/A-18 background of the three test pilots. All three test pilots had over 1,000 flight hours each in the F/A-18. The first evaluation on the F/A-18 HARV consisted of two flights flown by LT Dave Prater (USN) and focused primarily on flying qualities, pitch control margin/nose down pitch recovery capability and a limited look at tactical utility.

The second evaluation was completed in March of 1994 and focused on validating extensive ground based simulation on lateral directional control power requirements, high AOA flying qualities and tactical utility.

LT C. A. Sternberg (USN) and Capt Ricardo Traven (CAF) piloted the F/A-18 HARV for a total of eight test missions (7.1 flight hours) in one week (21 to 25 March, 1994) which was a new NASA F/ A-18 HARV record. LT Sternberg became the fifth pilot to ever fly the HARV and Capt Traven was the sixth.

\section{Background}

o November 1992:

-NASA/NAVY "HANG"

-Tactical Utility

-LT Dave Prater - 2 Flights

o March 1994:

-NASANNAVY "HAIRRY"

-Tactical Utility

-LT Chuck Sternberg - 4 Flights

-Capt. Ricardo Traven - 4 Flights 


\section{TEST OBJECTIVES}

The objectives of the flight test were:

(1) Provide in-flight validation of extensive ground-based simulation studies, including specific maneuver setup and execution, rating methodology and limited data comparison between flight test and simulator research.

(2) Assess overall handling qualities at high angle of attack (AOA) including motion effects on pilot orientation and spatial awareness, pilot cueing and pilot workload.

(3) Determine tactical utility benefits generated from the aircraft's thrust vectoring system.

Although the majority of flight test concentrated on simulation validation and test methodology verification, the pilots were able to take a limited look at the "tactical utility" of the HARV with thrust vectoring. The implementation of thrust vectoring on the HARV allowed the Navy to assess the implications of increased control power at high AOA on a current operational platform. Tactical utility testing consisted of conducting "canned" basic fighter maneuvers against a NASA F/A-18 target airplane. Both offensive and defensive maneuvers were evaluated.

\section{Objectives}

- Validate Simulation Test Methodology

-Maneuver Setup

-Limited Data Comparison

-Motion Cue Effects

- High AOA Handling Qualities

- High AOA Tactical Utility 


\section{FLIGHT PROFILS}

This evaluation was planned in four phases. The first flights, Phase I, concentrated on introducing the pilots to the HARV's handling qualities up to 70 deg AOA. Phase I maneuvers contained more quantitative closed loop maneuvers in order to expand the Navy's. High AOA data base and provide the pilots with an introduction to flight at High AOA. These maneuvers consisted of bank angle and heading captures, loaded rolls and constant thrust stabilized pushovers.

Phase II consisted of maneuvers that highlighted the lateral directional axes and evaluated roll performance and handling qualities during gross acquisition tasks. These maneuvers were developed by the test team during extensive simulation under the HAIRRY program and are commonly referred to as HAIRRY maneuvers. HAIRRY is an acronym for "High AOA Investigation of Requirements for Roll and Yaw" and is a joint U.S. Navy/NASA project which is part of NASA's High AOA Technology Program. The purpose of the HAIRRY program is to quantitatively identify/define lateral directional control power requirements at high AOA for incorporation into next generation aircraft design specifications. Data gathered included pilot comments, maneuver ratings, and dynamic response characteristics.

Phase III consisted of tactical/mission representative tasks to evaluate the overall tactical utility of thrust vectoring. These maneuvers were tactically oriented and were designed to minimize "pure" performance differences between the HARV and the target aircraft and provide repeatable engagements so that engagements flown with thrust vectoring on and off could be compared. For direct comparisons, the variations between setups needed to be minimized and the maneuvers were designed with that in mind. Tactically oriented maneuvers included: 1 v 1 canned setups against a predictable target, J Turns (Post-Stall Reversals), and Flat Scissors. The focus of these maneuvers was to assess how the pilots effectively used thrust vectoring in an "operational environment".

\section{Profiles}

o Phase I - HARV Fam

- Bank / Heading Captures

- Phase II - HAIRRY

- Lateral Gross Acquisition

- Offensive Loaded Reversals

- Defensive Rolls

- Phase III - Tactical Utility

- Flat Scissors

$-\mathrm{J}$ Turn

$-1 \mathrm{v} 1$ 


\section{HAIRRY}

Although the HAIRRY maneuvers were designed primarily for evaluating open loop roll performance and handling qualities during target capture, they provided some insight as to the tactical utility benefits of such a maneuver. The maneuvers were designed to gather quantitative data but were intentionally designed to be mission oriented. These maneuvers were designed to eliminate the longitudinal axis from the equation. The team wanted to focus specifically on the lateral directional axes. The maneuvers were also designed in such a way that they could be executed in flight the same way they were executed in the simulator. This allowed for a direct comparison of flight test and simulation data.

The three maneuvers used were the Lateral Gross Acquisition, Offensive Loaded Reversal and Defensive Roll. The Lateral Gross Acquisition and the Offensive Loaded Reversal maneuvers were initiated using a full lateral stick, step input (feet on the floor) followed by at least 130 to 180 deg of bank angle change, and ended with a capture of the target between vertical bars on HUD. This allowed the pilot to evaluate open loop roll performance as well as Handling Qualities during the capture portion of the task. The Defensive roll was basically on open loop roll performance task with no capture, but the pilot used an attacking aircraft to evaluate his ability to maintain situational awareness during the maneuver. During the simulation the maximum steady state roll rate and roll mode time constant were varied and each maneuver was performed over the full range of AOA's from 15 to $60 \mathrm{deg}$. In flight, the parametrics were not varied per se, but these maneuvers were flown first with the baseline flight controls (no thrust vectoring) and then flown with the thrust vectoring on (Research Flight Control Systems - RFCS, engaged). This allowed the pilots to evaluate the effects of a substantial increase in control power provided by the RFCS.

\section{HAIRRY Maneuvers}

Lateral Gross Acquisition
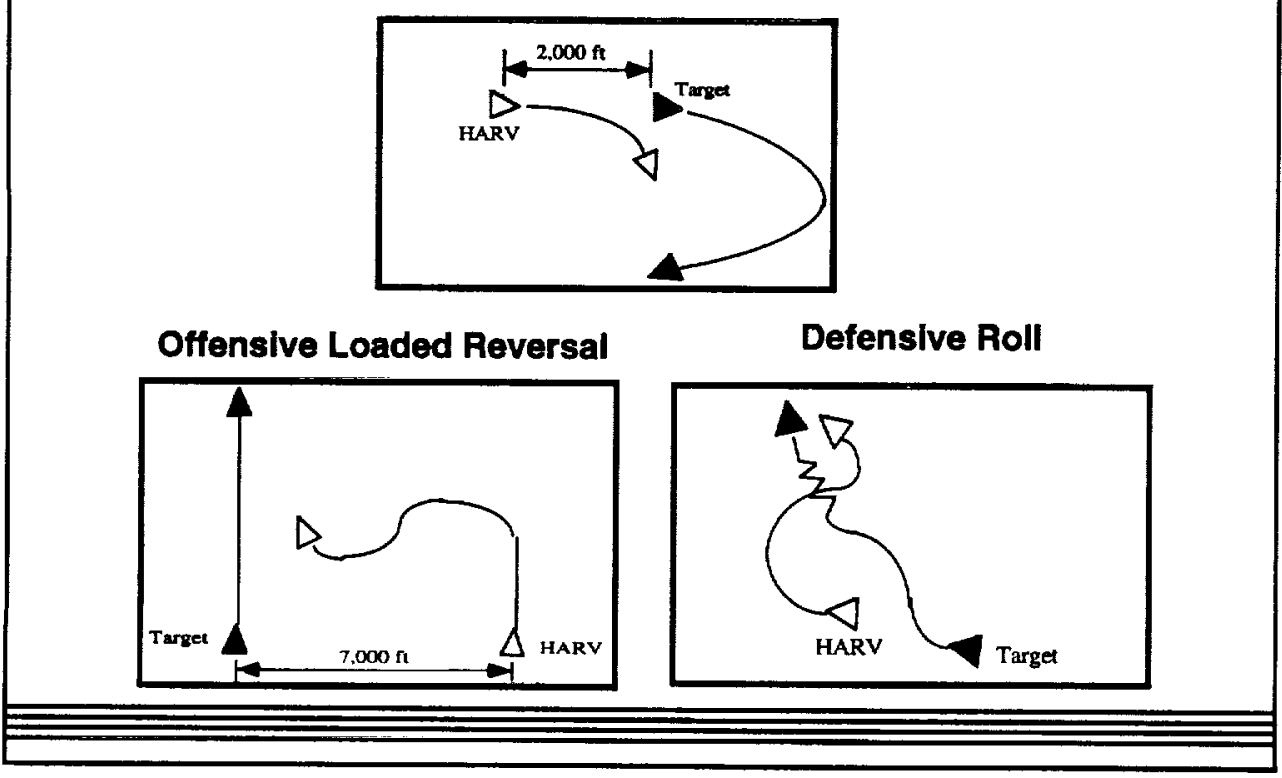

Page 5 


\section{OPERATING IN THE HIGH AOA ENVIRONMENT}

Tactical Maneuvering in the High AOA environment differs significantly between conventional aircraft (F/A-18/F-14) and Thrust vectoring/augmented aircraft (HARV).

In addition to the limited AOA achievable, "conventional" platforms are severely limited in the lateral directional axes at high AOA due to reduced control power and increased departure susceptibility. As a result, to utilize the lateral directional axis, the AOA must first be reduced to achieve acceptable performance. Once the AOA is reduced, the aircraft is rolled to reposition the lift vector, and then the AOA is increased as required for the tactical situation. In this manner, the axes are decoupled intentionally by the pilot to compensate for a control system that provides unsuitable lateral directional performance at High AOA.

It is in this manner the the HARV has an advantage over the conventional design in that the augmented control system provides the pilot with enhanced lateral directional performance at High AOA and the pilot is not required to decouple the axes manually. This opens up a whole new way of thinking and provides the pilot with an enhanced set of tools to prosecute the tactical situation..

In the past, it seems the general mindset about thrust vectoring was the ability to fly to $70 \mathrm{AOA}$, to pitch to 70 deg AOA. Much of the emphasis was all pitch and very little about rolling/yawing at those AOA's. During the HARV evaluation, the test pilots made a conscious and concerted effort to investigate the lateral directional axes from a tactical perspective.

\section{Lateral Directional Vs. Longitudinal}

Conventional:

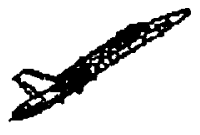

(1)
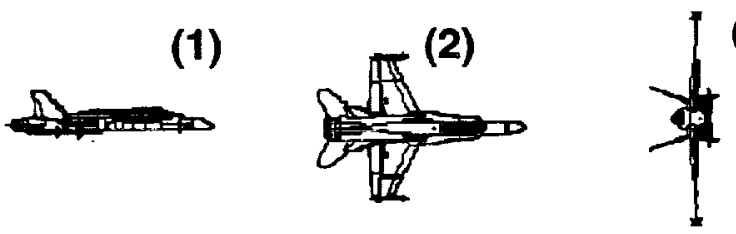

High AOA:

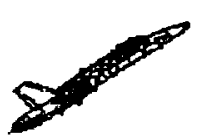

(1)
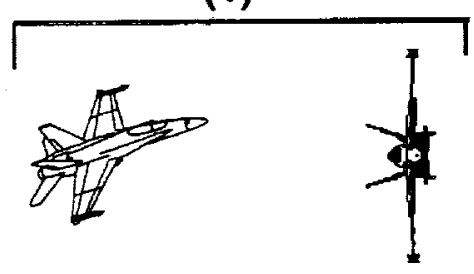

Page 6 


\section{ROLL PERFORMANCE CLASSIFICATION}

When a pilot executes a capture task designed to evaluate the lateral directional axes, the resulting comments are based on two aspects of the aircraft response - the roll performance during the bank angle change to get to the target and the handling qualities during the capture. The pilot bases his comments on roll performance on the initial roll response, perceived linearity of the roll response and roll rates, as well as time required to perform a given task. Handling qualities aspects deal with the ability to roll the airplane and then capture a target as in the gross acquisition task. The pilot's handling qualities comments deal not so much with roll performance, but on the predictability and controllability of the lateral directional axes in accomplishing the task. Looking at either issue separately can be misleading. The handling qualities might be superb, but the roll performance may be so poor as to be tactically useless.

Therefore, the test team decided it was imperative to separate roll performance issues form handling qualities issues. In order to "quantify" the pilots' comments on mission suitability/roll performance and separate handling qualities issues, the Roll Performance Classification (RPC) scale was developed by the Navy/NASA HAIRRY team. The RPC scale isolated maneuvering aspects only and thus could more directly correlate pilot ratings/comments with control power-related characteristics .

The RPC scale relates roll performance/lateral directional maneuverability to mission effectiveness. It is very task dependent and relies on the pilot's tactical experience to evaluate the roll performance. It was intentionally designed to be simple - The "Good, bad, ugly" scale. The beauty of this scale is in its simplicity. Roll performance which is evaluated as "Enhancing, tactically superior" is rated as a 1. Unacceptable roll performance is rated as a 4 . Tasks which exhibit roll performance between these two levels are broken out in the figure below.

\section{Connecting The Pilot w/ The Engineer}

\section{Roll Performance Classification}

\begin{tabular}{|c|c|c|}
\hline $\begin{array}{c}\text { RoIl Performance for } \\
\text { Mission Effectiveness }\end{array}$ & $\begin{array}{c}\text { Improvements In } \\
\text { Roll Performance }\end{array}$ & Numerical \\
\hline $\begin{array}{c}\text { Enhancing - } \\
\text { Tactlcally Superlor }\end{array}$ & None Warranted & 1 \\
\hline $\begin{array}{c}\text { Satisfactory - } \\
\text { Mlssion Requirements Met }\end{array}$ & $\begin{array}{c}\text { May be warranted, } \\
\text { but not required }\end{array}$ & 2 \\
\hline $\begin{array}{c}\text { Unsatisfactory - } \\
\text { Mlssion Requirements Not Met }\end{array}$ & Required & 3 \\
\hline $\begin{array}{c}\text { Inadequate - } \\
\text { Could Not Perform Maneuver }\end{array}$ & Mandatory & 4 \\
\hline
\end{tabular}




\section{ROLLING ABOUT THE VELOCITY VECTOR}

An important issue to consider when discussing tactical utility is whether the pilot is able to interpret and predict his flight path and or position his lift vector to capture a target, especially at very high AOA's. Will disorientation be a factor?

During flight test, one of the pilots came up with a "method" for describing how he was able to adapt to the high AOA environment and helps to describe the "AOA cone" for the folks on the ground.. It's called"Sterno's Lampshade on a Dog" high AOA visualization technique. The alpha cone to a HARV pilot is like a lampshade on a dog.

It is quite common for a dog to wear a "lampshade" or plastic cone on his head to keep from chewing stitches or whatever. In a very short period of time, the dog learns how to perform his daily routine with this lampshade on his head and he adapts quite quickly.

When a pilot straps on the HARV or any other high AOA vehicle, he is, in a sense, strapping on a lampshade, which is defined by his AOA. The pilot can visualize his "Lampshade" or ALPHA cone by sampling the aircraft's lateral directional response at a given AOA. The small perturbations describe a small arc and the pilot can extrapolate the dimensions of his cone based on that small cone segment. The pilot can visualize where this "Lampshade" is and can superimpose it on the outside world. The pilot may use his "lampshade" in the following manner. In a tactical scenario, the pilot knows where the target is, predicts his flight path and determines the best way to get the target to fly into his "lampshade" for the capture. Once the target has intersected the "lampshade" surface, the pilot can can use the lateral directional axes for the capture. Of course, there are some instances where this is not practical and a more conventional capture would be more efficient, but we'll show some examples where this kind of technique can be useful.

Utilizing the "lampshade" in the high AOA environment allows the pilot to modify his way of thinking about air-to-air engagements

\section{Sterno's Lampshade on a Dog High AOA Visualization Technique}

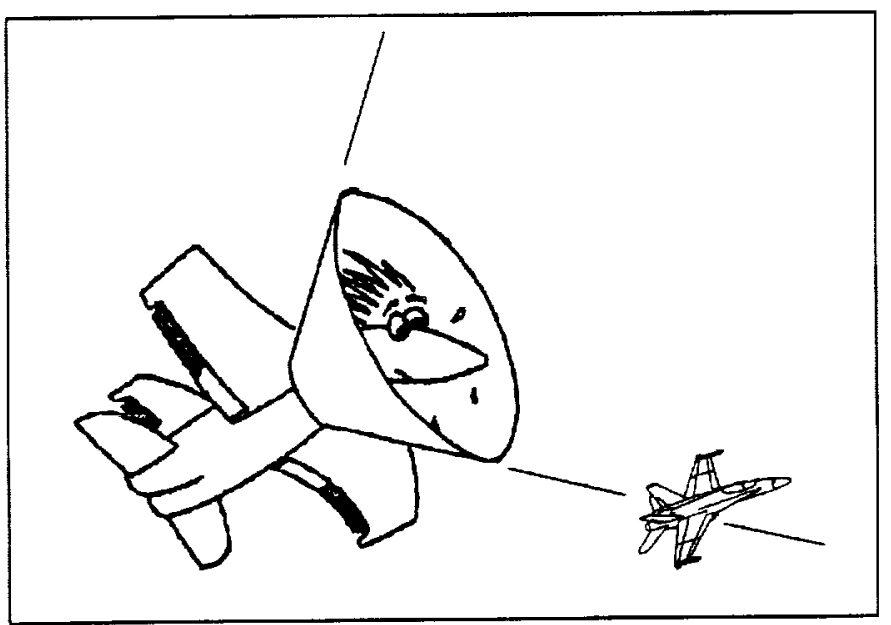

Page 8 


\section{TACTICAL UTILITY MANEUVERS}

Three maneuvers were used to look at tactical utility. These maneuvers were designed to eliminate a direct performance comparison and factor out thrust to weight effects. The HARV suffers dramatically from a thrust to weight standpoint and our purpose was not to evaluate the HARV's tactical utility, but evaluate the tactical utility of the thrust vectoring system. To accomplish this purpose, all maneuvers were flown first with the thrust vectoring disengaged, i.e. the baseline HARV airframe. The maneuvers were then repeated with the thrust vectoring system engaged.

The flat scissors maneuver emphasized the slow speed knife fight.

The J-turn was the test pilot's first look at a classic post stall reversal. It emphasized larger magnitude, more aggressive maneuvering using the entire alpha range of the aircraft. It was imperative that the test pilots learned and understood the post stall reversal prior to moving on the the $1 \mathrm{v} 1$ maneuver.

The $1 \mathrm{v} 1$ maneuver was more of a free form exercise which was initiated from fixed initial conditions. This maneuver allowed the pilot the freedom to maneuver the aircraft as he saw fit to prosecute and capture the target in the shortest amount of time. It was basically an open forum where the pilot could experiment with the added capability provided by the HARV and discover/exploit those regions. After the $1 \mathrm{vl}$ was flown with the RFCS on and off, the HARV was given a 2,000 ft altitude advantage at the merge, which was increased by $2,000 \mathrm{ft}$ for subsequent maneuvers with a maximum split of $6,000 \mathrm{ft}$.

During the J-turn and $1 \mathrm{v} 1$ Maneuvers, the targets flew a prebriefed flight path so that a direct comparison could be made between maneuvers flown with thrust vectoring on and off.

\section{Tactical Utility Maneuvers}

\section{Flat Scissors}

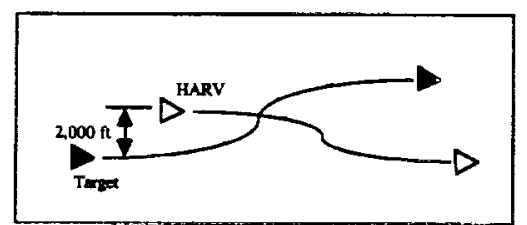

1v1 w/Predictable Target
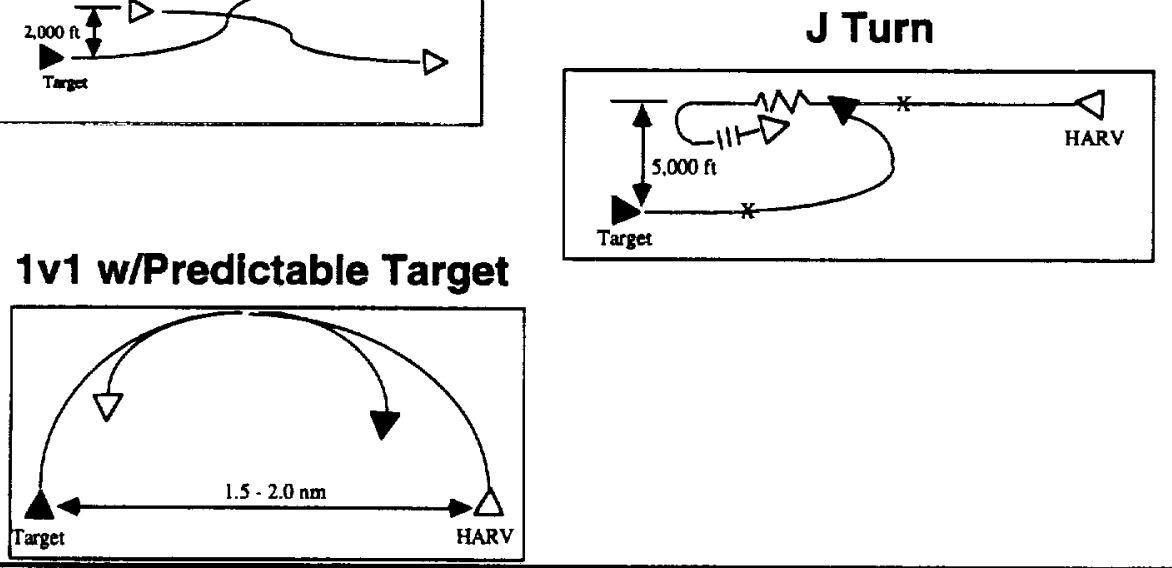

Page 9 


\section{FLAT SCISSORS}

The Flat Scissors forced a slow speed knife fight at close quarters from a neutral setup. In this type of fight, lateral directional maneuverability and the ability to fly slower than one's opponent is critical.

When flown with the baseline HARV, the HARV was unable to achieve any tactical advantage over the chase Hornet. The flying qualities and lateral directional maneuverability of the HARV were very poor and evaluated as "unsuitable" for the mission, RPC 3.

With the thrust vectoring engaged, the HARV had a clear and distinct advantage over the target aircraft. It was a night and day difference. The HARV was able to achieve a tactical advantage almost immediately by exploiting the ability of the HARV to fly much slower/higher AOA than the target aircraft and maneuver at will while maintaining that high AOA. Another advantage enjoyed by the HARV was to ability to maneuver much more effectively in the lateral directional axes at Clmax and below. By being able to maneuver aggressively in the lateral directional axes at $35 \mathrm{AOA}$, where the target aircraft was flying, the HARV was able to quickly obtain an offensive advantage by early turning and maneuvering aggressively to reposition behind the target aircraft without using extremely high AOA range.

The thrust vectoring capability of the HARV provided it with a clear and distinct advantage over the baseline F/A-18 with similar thrust to weight. The superior lateral directional maneuverability at Clmax and below, and the ability to fly and maneuver at extreme AOA's is a distinct tactical advantage in the slow speed arena.

\section{Thrust Vectoring - Flat Scissors}

- Downrange Travel Reduction

- Superior Maneuverability At All AOA's

- Effective, Offensive Repositioning

- Immediate Offensive Advantage From Neutral

- Clear Distinct Advantage Over Baseline F/A-18 


\section{J TURN / POST STALL REVERSAL}

The J-turn/Post Stall Reversal was used both as a training maneuver and a tactical maneuver. It was used initially to introduce the pilots to the techniques required to execute this unique maneuver. Once this was accomplished, the pilots evaluated the tactical utility of the maneuver in acquiring a target. Both pilots found it particularly advantageous in prosecuting a target provided that a large altitude advantage was enjoyed.

The setups worked out such that the pilot pitched to 50 to $60 \mathrm{deg}$ AOA, then when the target had "entered the lampshade", 4,000 ft below and offset laterally, a pure lateral directional capture was performed using full lateral stick. The HARV ended up directly overhead the target 60 to 90 deg nose down. It was flown as a high to low, vertical capture. The pilot had to time the lateral input depending on the lateral separation and slant range of the target. Even with all those variables, the pilots were able to perform successful captures after only a couple of attempts. This was in indication that the pilots were able to adapt rather quickly to this new environment.

Both pilots agreed that this maneuver was very dependent on the initial setup and that it could only be used in very specific instances during a fight when the pilot had the required separation and altitude advantage. This maneuver was very dependent on a high to low capture for tactical usefulness .

One situation the pilot must be careful to avoid is the blind lead turn. There were many cases where it would have been advantageous to begin the maneuver, but it had to be delayed in order to ensure clear view of the target throughout the maneuver. This is an issue that will become more critical as more aircraft are capable of high AOA flight.

Being able to perform this maneuver is a distinct tactical advantage when used properly. It was evident from this maneuver that if used incorrectly or at the wrong time it could be particularly disadvantageous due to the predictability and large altitude/ energy losses involved.

\section{Thrust Vectoring - $\mathbf{J}$ Turn}

o Exploits Lateral Directional Capability $50-60{ }^{\circ} \alpha$

- Setup Critical

- High To Low Distinct Advantage

- Capturing Directly Above Target

o Blind Lead Turns

o Altitude and Geometry Requirements

o Not A Cure All

Page 11 
The 1v1 was an effective maneuver for evaluating not just the capabilities of the HARV but evaluating the "capability of the pilot" to use the increased control power to his advantage. Because the pilot was able to prosecute and capture the target in any way he saw fit, the test team was able to evaluate the capability of the pilot to use the added capabilities of the HARV.

The HARV was allowed to maneuver at will after a 180 out pass with the target. The target performed a $300 \mathrm{kt}, 3 \mathrm{~g}$ level turn until the HARV made the capture. With thrust vectoring off, the HARV executed a max power, energy sustaining, nose low, slice turn back towards the target at corner speed using 4 to $4.5 \mathrm{~g}$. The target was captured from low to high using an aggressive, energy dissipating pull to 35 AOA. During this maneuver, very little roll rate was commanded, and time to capture was based primarily on maximum turn performance at corner speed, with a maximum pitch rate command to capture. The initial plan for the thrust vectoring on capture was to fly the first part of the capture the same, but initiate the pitch capture much earlier by exploiting the capability of the HARV to pitch to 70 deg AOA. During the simulation this did not decrease the time to capture dramatically. There had to be a better way. Instead of trying to exploit the longitudinal axis, why not exploit the lateral directional axes? It was decided to utilize the superior lateral directional capability of the HARV and the "lampshade" technique for the capture.

At the merge, instead of digging nose low back towards the target, the velocity vector was "cast" up at the merge, like the beginning of the J-turn and eased back toward the target in one circle flow. The HARV then delayed "on the perch" at mid range AOA ( $35-45$ degrees) to conserve energy and predicted the targets flight path. At this point an altitude advantage had been gained and lateral separation had been achieved.. When the target flew into the HARV's lampshade, a pure lateral directional capture was executed, high to low.

\section{Target Capture During 1v1}

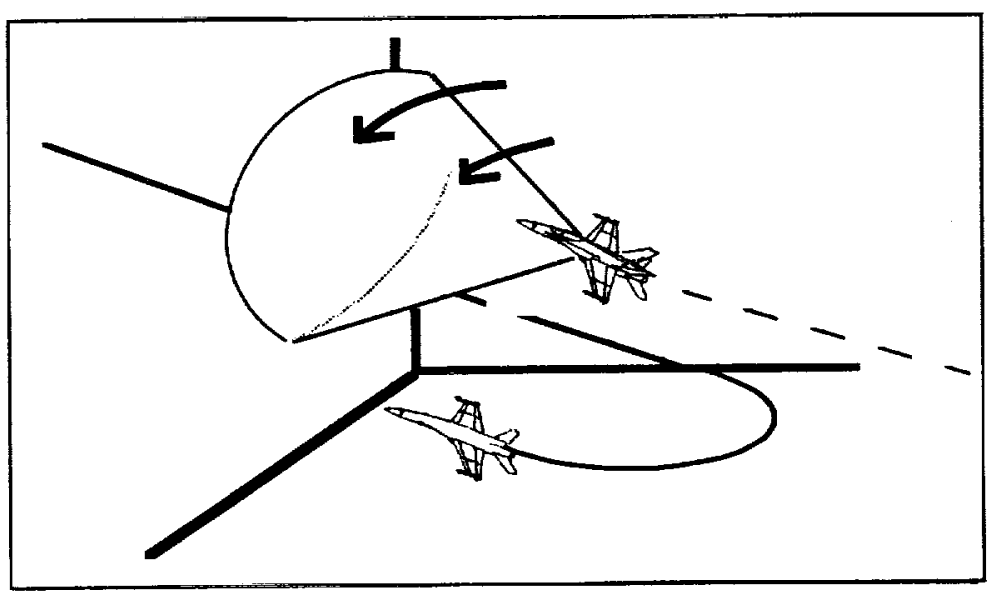

Page 12 


\section{1v1 CONCLUSIONS}

The 1v1 was flown multiple times by both pilots, and different techniques were utilized as described earlier. Not all 1v1's worked out as the pilots had planned, but some useful conclusions were drawn from even the less than successful captures.

1. Using the capability of the HARV to roll at high AOA's is just as tactically advantageous as being able to pitch to $70 \mathrm{deg} A O A$. In some cases, it was even more unpredictable from a target's perspective and should not be discounted. An increased emphasis on utilizing the lateral directional axes is warranted.

2. An altitude/energy advantage is even more critical when maneuvering beyond Clmax than it is in conventional aircraft due to the high energy losses experienced at high AOA. The post stall capability must be used judiciously and carefully for it to be effective.

3. Both pilots adapted quickly to this new environment and were able to use the increased capabilities of the HARV to a tactical advantage. It's not too hard.

4. After using the post stall capabilities of the HARV, energy addition is critical and emphasizes the need for bigger motors and lighter systems.

\section{Thrust Vectoring - 1v1}

o Pitch Not Always The Answer

o Adaptability Evident, Modified J Turns

- Altitude Advantage Effects

○ Prudent Use Of Flight Beyond $\mathrm{C}_{\mathrm{Lmax}}$

o High Energy Requirements 


\section{ROLL PERFORMANCE CLASSIFICATION}

The Roll Performance Classification proved to be simple and useful during flight test. The simplicity was a benefit, especially since between maneuvers the pilot also had to provide comments and HQR's on the capture. The RPC's given for the maneuvers were consistent between pilots and correlated nicely with pilot comments. It was evident that the RPC was very maneuver specific and one must be careful not to correlate RPC's will roll performance data without identifying the maneuver. For instance, during a flat scissors, where very little roll rate was commanded, for that task, $10 \mathrm{deg} / \mathrm{sec}$ might have been tactically superior but that same roll rate during a guns defense would have been unacceptable. Interpilot data was consistent for the same maneuver.

The pilots emphasize that although simple, the RPC scale does help to quantify pilot comments and tactical utility. The scale can be used in a general sense for any maneuver, since specific performance criteria is not identified. It is good method to help to evaluate tactical utility when no other quantitative data is available.

The RPC provided consistent results and correlated fairly consistently with simulator data.

When RPC ratings are used in conjunction with HQR's, regions of acceptable handling qualities and performance can be identified. This scale also allowed the pilot to interpret the tactical situation and rate lateral directional performance based on his own background, knowledge and tactical experience.

\section{RPC Scale}

- Simple, Effective, Easy To Use

- Maneuver Specific

- Consistent Results

- Allows Pilot Interpretation Of Tactical Situation

Page 14 


\section{RPC AND TACTICAL UTILITY}

The RPC was also useful as a another measure of tactical utility. RPC ratings for maneuvers flown with the baseline HARV were all 3's and 4's. This was in the unsuitable, unacceptable range for roll performance. Flying identical maneuvers with the thrust vectoring engaged, resulted in RPC ratings in the 1 to 2 range which is the "suitable" and "tactically superior" range. It was clear from the pilot comments during both the HAIRRY maneuvers and the tactical maneuvers that the thrust vectoring provided the pilot with increased tactical utility. These pilot comments backed up and correlated nicely with the RPC ratings. Again, the beauty of the RPC scale is that it has the flexibility to be used for almost any task and gives the pilot the ability to rate the maneuver based on his perception of the tactical utility.

\section{Maneuver Summary}

\begin{tabular}{|c|c|c|c|}
\hline Maneuver & $\overline{A O A}$ & $\begin{array}{l}\text { RPC } \\
T V \text { Off }\end{array}$ & $\begin{array}{l}\text { RPC } \\
\text { TV On }\end{array}$ \\
\hline \multirow[t]{4}{*}{ Lateral Gross Acquisition } & 15 & - & 1 \\
\hline & 30 & 3 & 2 \\
\hline & 40 & $\cdots$ & 2 \\
\hline & 60 & $=$ & 2 \\
\hline \multirow[t]{4}{*}{ Offensive Loaded Reversal } & 15 & $=$ & 1 \\
\hline & 30 & 4 & 2 \\
\hline & 45 & - & 2 \\
\hline & 60 & $=$ & $\overline{2}$ \\
\hline \multirow[t]{3}{*}{ Defensive Roll } & 30 & 3 & 2 \\
\hline & 45 & - & $\overline{1}$ \\
\hline & 60 & $=$ & 2 \\
\hline Flat Scissors & 30 & 3 & $\overline{1}$ \\
\hline J Tum & $\bar{A} / \mathbf{R}$ & - & 2 \\
\hline 1 v1 Level & $A / R$ & - & $\overline{1}$ \\
\hline 1v1 6K' Split & $A / R$ & - & 2 \\
\hline
\end{tabular}

Page 15 


\section{PILOT ADAPTABILITY / SPATIAL AWARENESS}

One of the key components of the tactical utility equation and one that must not be left out is the pilot. The pilot interprets the tactical situation and decides what actions must be taken to achieve the desired outcome. Regardless of what capability the engineers have given his airplane, his knowledge and more importantly his perception of his own capabilities will affect his actions. Is the pilot's perception of what is happening close to reality? When a pilot commands roll and perceives the aircraft yawing, will that create a spatial awareness problem? Are his perceptions influenced by the dynamics of the task and the problem of prosecuting a bogey?

One of the purposes of this flight test was to find out what the pilot actually perceives and how he interprets the situation, specifically at high AOA. Both pilots had extensive simulator time at high AOA, but how would actual flight test affect his comments on tactical utility and spatial orientation.

After eight flights, numerous closed loop, open loop and tactical tasks, both pilots were of the same opinion - "This is great, it's just like any other kind of flying." "Easy to use, feels good." "It's intuitive, even at extreme AOA's, the coning effect was not disorienting in any respect." "No surprises". Keeping in mind, that this was both pilot's first experience with flight at those extreme angles of attack, up to 70 degrees, these comments are poignant. Neither pilot ever felt disoriented. Neither pilot felt any significant sideforces and each pilot adapted very quickly to high AOA flight. Both pilots were very surprised that it felt so natural. They had expected some disorientation or at least some confusion, especially when "coning" around at 70 deg AOA.

Both pilots learned how to use the high AOA capability of the HARV very quickly. After only a few tactical maneuvers, pilots were flying the HARV very efficiently and minimizing time to kill during the $1 \mathrm{vl}$ 's. Both pilots were able to factor the capabilities of the HARV into their tactical plan real time and successfully maneuver the aircraft. It was evident that flight at extreme angles of attack will pose no more of a training problem in the fleet than normal ACM training. It's just another tool that the fleet pilot needs to learn how to use.

\section{Pilot Adaptability}

- High AOA Flight Regime Adaptability

○ Good Simulator Fidelity

o No Disorientation

o Intuitive, Felt Natural

- No Extensive Training Required

(If Ricardo could figure it out ...)

Page 16 


\section{DEPARTURE RESISTANCE}

A tremendous tactical benefit from the thrust vectoring system on the HARV is the superb departure resistance that it enjoys. For many years, departures, subsequent out-of-control flight and even aircraft and aircrew losses have been a somewhat regular occurrence in the ACM business. Some solutions have been limitations to the aircraft in terms of AOA but in the HARV we have increased departure resistance by expanding the aircraft envelope.

Throughout all eight flights, there were no departures and at no time did the pilot even approach the departure boundaries. This is a significant point given that these maneuvers were targeting the edge of the current HARV envelope using abrupt, full stick, long duration inputs.

A departure during an ACM engagement is usually followed by the departing aircraft getting gunned. Departure resistance is a significant tactical advantage. This is an aside that is often overlooked. This allows the pilot to spend less time worrying about dancing around the edge of the envelope and more time flying right on the edge if required.

\section{Departure Resistance}

- No Departures Experienced

o "Carefree" Maneuvering

o Concentrate On Task At Hand

- Definite Tactical Advantage

o Huge Benefit - Safety Standpoint 


\section{LATERAL STICK VERSUS RUDDER PEDALS AT HIGH AOA}

There are two basic schools of thought on how the lateral-directional axes should be controlled at high AOA. One school of thought espouses the "Classical" theory that flight control laws should be written with pilot perceived motion as the driver. i.e. Yawing motion at high AOA should be controlled by rudder pedals and roll should be controlled by lateral stick. The proponents of this theory argue that it would be disorienting to the pilot to input lateral stick at $60 \mathrm{deg} \mathrm{AOA}$ and perceive almost a pure yawing motion, the coning effect.

The other side of the argument we'll call the "Feet on the floor"' guys. This school of thought believes in simplicity such that at high AOA's, lateral stick controls all lateral directional motion with the flight controls coordinating sufficiently not to warrant use of rudder pedals.

Most high AOA simulation efforts utilize feet on the floor control laws for interpilot and intrapilot data consistency Both Navy pilots have been involved in many feet on the floor simulations and one was a proponent of the classical method and one was a feet on the floor guy.

After having flown the HARV both pilots are firm in their belief that flying "feet on the floor" at high AOA is quite natural, easily adaptable and poses no spatial awareness or disorientation problems. Other than providing consistent results for test missions, it is very simple, and simplicity when flying at high AOA's is key.

A compromise for both parties would have the rudder pedals and lateral stick command the same control system response at high AOA. In this way, a pilot would be able to use what feels comfortable to him. The only difficulty would be that at lower AOA's, it is desired to have rudder pedals command yaw and lateral stick command roll. There needs to be a crossover AOA below which rudder pedals and lateral stick behave in a "conventional" sense. Currently in the F/A-18, the crossover AOA where the rudder pedal to rolling surface interconnect comes into play is 13 deg AOA.

\section{Rudder Pedal vs. Lateral Stick}

o Pilot Preferences

- Keep it simple

- Compromise/Solution

- Crossover AOA 


\section{CONCLUSIONS}

The test team is convinced that the additional control power provided to the HARV by the thrust vectoring system is tactically advantageous to the fighters of the future in terms of providing the pilot with an "enhanced" set of tools to enter the fight with.

Both pilots were able to experience the tactical benefits that thrust vectoring provided them on the HARV. The increased control power was quite impressive at the extreme angles of attack ( $45 \mathrm{deg}$ and above) and the ability to fly and maneuver at the extreme AOA's proved superior in a slow speed flight. The thrust vectoring also provided a significant capability when operating at or just below CLmax, (35 deg AOA in the F/A-18). In some cases this added capability at moderate AOA's was more tactically useful that the ability to fly at $70 \mathrm{deg} \mathrm{AOA}$. The pilots found that only momentary excursions to 60 to $70 \mathrm{deg} \mathrm{AOA}$ were practical due to the large energy losses experienced at these AOA's.

The increased control power allowed the HARV pilot to maneuver much more effectively within the current Hornet envelope and maneuver at will outside the envelope, resulting in a clear and distinct tactical advantage.

The pilots were pleasantly surprised at the tactical utility of the lateral directional maneuverability at all AOA's. They realized that although the ability to pitch to extreme AOA's, it is also a significant capability to command significant roll/yaw rates at those AOA's.

Both pilots concluded that the full potential of the thrust vectoring system would not be realized until a much higher thrust to weight ratio is achievable on the test platform. The large energy losses experienced during high AOA maneuvering requires more thrust, quicker energy addition - We need bigger motors and lighter, integrated systems.

Finally, thrust vectoring is not a cure all in the tactical environment, but it adds some tremendous benefits to the fighter pilot's bag of tricks and it is essential to have this capability in fighters of the future.

\section{Conclusions}

o Benefits At Extreme AOA's

- Lateral Directional Maneuverability/Repositioning

- Pitch Pointing/Intimidation

o Judicious Use

- Large Energy Dissipation Post Stall

- Altitude/Energy Advantage Critical

- Emphasizes Need For Bigger Motors/Lighter Systems

o Benefits Inside Current Envelope

$-\leq \mathrm{C}_{L \max }$

o Not A Cure All

- Powertool For Your Toolbox

Page 19 
I I 1 